\title{
Blanqueamiento dental en niños y adolescentes. ¿El epílogo de un mito? Revisión de la Literatura
}

$\begin{array}{r}\text { María Gabriela Acosta de Camargo, }{ }^{1} \text { (i) } \\ \text { Alfredo Natera, } \\ \text { Mónica Rodríguez, } \\ \text { Eva Pimentel, } \\ { }^{2} \\ \text { María Begoña Tortolero, }{ }^{2} \\ \hline\end{array}$

\section{Resumen}

Introducción: En torno al blanqueamiento dental existen muchas afirmaciones con poco soporte científico, tales como: los blanqueamientos son ácidos, dañan el esmalte, se utilizan férulas, hay restricción en dieta y en la gestación, con límite mínimo de edad para su uso, entre otros. Para la odontología es de suma importancia que toda afirmación se realice responsablemente con una evidencia científica para entregar lo mejor de cada tratamiento a los pacientes. Objetivo: Conocer las posibles indicaciones del blanqueamiento dental en niños y adolescentes, modalidades, los efectos sobre los tejidos blandos y duros, así como sus efectos secundarios, la hipersensibilidad dentinaria, su tratamiento farmacológico y su impacto psicosocial. Materiales y métodos: se realizó una búsqueda electrónica de los siguientes buscadores: PubMed, LILACS, SCIELO, Cochrane Library y SCOPUS, limitando la búsqueda entre los años 1998 y 2020 utilizando los términos relacionados a la investigación. Se incluyeron 71 artículos que cumplieron con los criterios de inclusión. Resultados: la mayoría de los autores recomiendan el uso de sistemas de blanqueamiento dental de acuerdo con parámetros establecidos.

Palabras clave: blanqueamiento dental, niños, adolescentes.

Artigo da revisao

\section{Clareamento dentário em crianças e adolescentes. O epílogo de um mito? Revisão da literatura}

\section{Resumo}

Introcução: No que diz respeito ao clareamento dos dentes, existem muitas afirmações com pouco apoio científico, como: o clareamento é ácido, danifica o esmalte, são utilizadas talas, há restrição de dieta e gravidez, com limite mínimo de idade para seu uso, entre outros. Para odontologia, é de extrema importância que

${ }^{1}$ Universidad de Carabobo.

${ }^{2}$ Universidad Central de Venezuela. 
todas as declarações sejam feitas de maneira responsável com evidências científicas para oferecer o melhor de cada tratamento aos pacientes. Objetivo: Conhecer as possíveis indicações para clareamento dental em crianças e adolescentes, modalidades, efeitos sobre os tecidos moles e duros, bem como seus efeitos colaterais, hipersensibilidade dentária, tratamento farmacológico e impacto psicossocial. Material e métodos: Foi realizada uma busca eletrônica nos seguintes mecanismos de busca: PubMed, LILACS, SCIELO, Cochrane Library e SCOPUS, limitando a busca entre 1998 e 2020, utilizando os termos relacionados à pesquisa. Setenta e um artigos que atenderam aos critérios de inclusão foram incluídos. Resultados: A maioria dos autores recomenda o uso de sistemas de clareamento dental de acordo com os parâmetros estabelecidos.

Palavras-chave: clareamento dental, crianças, adolescentes

Article of revision

\section{Teeth bleaching in children and adolescents. The epilogue of a myth? Literature Review}

\begin{abstract}
Introduction: Regarding teeth bleaching, there are many statements with little scientific support, such as: whitening is acidic, damages the enamel, guards are used, there is a restriction in diet and pregnancy, with a minimum age limit for its use, between others. For dentistry it is of utmost importance that all statements are made in a responsible manner with scientific evidence to provide the best of each treatment to patients. Objective: to know the possible indications for teeth bleaching in children and adolescents, the modalities, the effects on soft and hard tissues, as well as its side effects, dental
\end{abstract}

\section{Introducción}

El color de los dientes y la apariencia estética son factores que adquieren cada día mayor importancia en la sociedad actual. Las demandas estéticas exigidas hypersensitivity, its pharmacological treatment and its psychosocial impact. Material and methods: An electronic search of the following databases was performed: PubMed, LILACS, SCIELO, Cochrane Library and SCOPUS, limiting the search between 1998 and 2020 using the terms related to the research. Seventyone articles that met the inclusion criteria were included. Results: Most authors recommend the use of teeth whitening systems according to established parameters.

Keywords: teeth bleaching, children, adolescents.

a los profesionales de la odontología comienzan a edades cada vez más tempranas y son motivo frecuente de consulta. Suele recomendarse que algunos tratamientos estéticos, tales como el blanqueamiento dental, se difieran 
hasta los 18 años de edad, sin embargo, no parecen existir razones sustentables que justifiquen dicha limitación. Son muy escasos los estudios realizados en el grupo etario de los adolescentes en relación con este tópico. Los niños $\mathrm{y}$ adolescentes que tienen defectos de esmalte a menudo refieren dificultades para sonreír y también reportan problemas de autoestima. Esta población debe recibir atención profesional integral que incluya la identificación de su condición $\mathrm{y}$ tratamientos accesibles que mejoren su calidad de vida. Adicionalmente es importante señalar que no existe control en el acceso a una gran variedad de productos de blanqueamiento dental vendidos en anaqueles sin prescripción ni orientación por parte del especialista, lo que indica que la población adolescente ciertamente utiliza este tipo de agentes blanqueadores, sin la adecuada e indispensable supervisión profesional.

Las razones anteriormente expuestas han motivado a los autores a realizar una exhaustiva revisión de la literatura para evaluar la evidencia científica disponible en el tratamiento de blanqueamiento dental en niños y adolescentes, su bioseguridad, sus limitaciones, los efectos secundarios, las indicaciones de acuerdo a las necesidades del paciente y su uso posible cuando están presentes requerimientos como los defectos de esmalte. Además de destacar el alcance de los blanqueamientos y efectividad en niños y adolescentes, así como edades recomendadas para su uso.

Son variadas las razones que parecen justificar el uso de blanqueamientos en niños y adolescentes si los casos han sido estudiados, correctamente analizados, indicados y bajo un basamento científico y actualizado. El clínico debe conocer lo que es normal en la dentición, así como los beneficios a nivel psicosocial que pueden impactar la vida de un niño o adolescente con limitaciones para hablar o sonreír. Es imperativo estudiar los beneficios que brindan productos disponibles en el mercado como el peróxido de carbamida en contraposición al peróxido de hidrógeno.

\section{Materiales y métodos}

Para esta revisión de la literatura se realizó una búsqueda electrónica inicial a través de los siguientes buscadores: PubMed, LILACS, SCIELO, Cochrane Library y SCOPUS, desde el año 1998 hasta 2020 utilizando los términos: "Dental bleaching children", "dental bleaching adolescents", "tooth whitening adolescents", "analgesic", "anti-inflammatory", "dental bleaching", "dental sensitivity", "developmental enamel defects", "emotional impact", en inglés. Blanqueamiento dental en niños, Blanqueamiento dental en adolescentes, analgésicos, antiinflamatorios, blanqueamiento dental, sensibilidad dentaria, defectos del desarrollo del esmalte, en español.

De toda la búsqueda se obtuvieron 527 artículos, de los que se seleccionaron 72 artículos que cumplían con los criterios de inclusión, estos fueron: Revisiones sistemáticas, meta-análisis, estudios de casos y control, transversales y reportes de casos. También fueron incluidos aparte de estos artículos, recomendaciones de cuatro asociaciones dentales muy reconocidas de varios países. Se excluyeron los resultados con información irrelevante para la 
investigación, seleccionando solo los que tenían relación directa con el objetivo de la misma, con resumen disponible y acceso al artículo completo en los idiomas inglés y español. Se excluyeron comunicaciones de periódicos, conferencias, memorias de congresos, comentarios personales o editoriales. Finalmente la evaluación fue realizada por 2 revisores de acuerdo a los criterios anteriormente expuestos.

\section{Resultados}

De los 72 artículos que cumplieron con los criterios de inclusión, 61\% fueron de los últimos 5 años. En su mayoría los autores recomiendan el uso de sistemas de blanqueamiento dental de acuerdo con parámetros establecidos.

Pigmentaciones extrínsecas e intrínsecas del esmalte dental. Pigmentos propios del niño y del adolescente.

Los factores que influyen en el color de los dientes pueden ser genéticos, congénitos, metabólicos, químicos, infecciosos y ambientales. ${ }^{1}$ Las pigmentaciones extrínsecas están relacionadas al consumo de bebidas alcohólicas, té, gaseosas y café, así como suplementos de hierro, ${ }^{2}$ alimentos y biopelícula cromógena, higiene bucal deficiente entre otros. En niños en dentición mixta es frecuente la queja relacionada al color más amarillo que presentan los dientes permanentes cuando son comparados con los dientes primarios..$^{2-3}$

Existen manchas extrínsecas que se caracterizan por puntos o líneas oscuras discretas en la superficie del diente. La relación entre la microbiota oral en la biopelícula de niños y por las mismas sigue siendo objeto de estudio ya que posiblemente se relacionen en la dentición primaria4. Se ha reportado que las bacterias Gram-positivas, especialmente Actinomyces israelii y Actinomyces naeslundii, son los microorganismos cultivables predominantes de este tipo de pigmentaciones. $^{5}$ Así mismo, al encontrarse Actinomyces spp, se consigue una probabilidad cuatro veces mayor de manchas extrínsecas en comparación con los sujetos sin estas bacterias. ${ }^{6}$ Actualmente se ha confirmado la existencia de hierro en las manchas extrínsecas mediante espectrometría de masa de plasma acoplado inductivamente. Fue la primera vez que se accedió a los genes funcionales de las bacterias en la mancha negra y se encontraron los genes asociados con el hierro. ${ }^{7}$ Así se encuentra una bacteria que produce pigmentos, el B. melanogenicus, que actúa sobre las sales ferrosas de los alimentos y precipita óxido ferroso que se deposita sobre los dientes dando una coloración negruzca. Es una pigmentación que no tiene nada que ver con el tabaco. Afecta a las caras vestibulares y palatinas y se forma un anillo más cercano a la parte cervical del diente, es de fácil eliminación, pero recidiva con facilidad.

Otros pigmentos se originan de bacterias cromógenas que se encuentran en la boca, los más frecuentes son los bacilos piociánicos, que producen un pigmento que se llama fenacina y da coloración verde a los dientes. La pigmentación verde es muy frecuente en adolescentes, afecta a los incisivos superiores con más frecuencia y a nivel del cuello del diente, es de fácil eliminación, suelen recidivar y desaparecen espontáneamente con los cambios hormonales de la pubertad.

En relación a las pigmentaciones 
intrínsecas es necesario señalar que en contraste con el hueso, el esmalte y la dentina no se remodelan. Por lo tanto, los trastornos en la función de los ameloblastos y/o de los odontoblastos durante el desarrollo dental dan lugar a defectos permanentes, ya que una vez formado, el esmalte dentales un tejido acelular que no tiene capacidad reparadora, salvo el efecto protector y remineralizador de la saliva durante la etapa posteruptiva. Los defectos de desarrollo del esmalte (DDE) se consideran desviaciones de la apariencia normal de este tejido resultantes de una disfunción en el órgano dentario y pueden ser clasificados en dos grandes categorías: la hipoplasia de esmalte y la hipomineralización del esmalte. ${ }^{8-10}$

La hipoplasia del esmalte se considera un defecto cuantitativo, producto de alteraciones durante la fase secretora del esmalte. Clínicamente se puede observar como la ausencia parcial o total del esmalte de la superficie de la corona dentaria. Por otro lado, la hipomineralización del esmalte se considera un defecto cualitativo que resulta de una deposición deficiente del contenido mineral durante la fase de calcificación. El esmalte hipomineralizado presenta un grosor normal, con un cambio en su translucidez y la presencia de opacidades de color blanco, amarillentas o marrones. ${ }^{8,11-12}$

Los DDE ocurren a consecuencia de una injuria local o sistémica durante el proceso de odontogénesis. El traumatismo dentario y los procesos infecciosos en la dentición primaria pueden afectar el desarrollo de los dientes sucesores, así mismo cuando el trauma ocurre en la dentición permanente puede causar cambios de color irreversibles asociados a calcificaciones y pérdida de la vitalidad celular. ${ }^{2}$ Por otro lado, las opacidades pueden derivarse de alteraciones de origen sistémico como la hipomineralización molar-incisivo (HMI) y la fluorosis dental, así como de alteraciones genéticas como la amelogénesis imperfecta.

Así mismo se requiere enfatizar que las consecuencias de la pigmentación plantean un impacto significativo en el bienestar psicosocial de los niños, existen estudios que informan que pueden sentirse cohibidos, tener baja autoestima y evitar sonreír o socializar. ${ }^{13,14}$ Tanto jóvenes como niños en edad preescolar y primaria ya son conscientes de sus propias diferencias notables y pueden ser juzgados negativamente por sus pares. ${ }^{15,16}$ Una sonrisa atractiva juega un papel importante en la percepción general de la apariencia física. Esta demanda pública de una sonrisa más blanca y una estética mejorada ha hecho que el blanqueamiento dental sea cada vez más popular. En consecuencia, los padres y los medios de comunicación demandan información sobre el blanqueamiento dental para niños y adolescentes. ${ }^{17}$

Blanqueamiento dentalen niños y adolescentes.

El primer protocolo para la población joven fue publicado en 1994, sin embargo, la mayoría de las investigaciones en relación con los protocolos de blanqueamiento dental han sido realizadas en adultos. Este tratamiento suele ser una alternativa razonable en niños y adolescentes con pigmentaciones oscuras y opacidades relacionadas a defectos del desarrollo dental, ya que se considera el procedimiento más conservador pues no requiere desgaste o pérdida de tejido. ${ }^{3}$ 
Tradicionalmente se han utilizado dos técnicas: en el consultorio dental con geles a base de peróxido de hidrógeno $y$, ambulatorio, con geles a base de peróxido de carbamida, pero tienen diferentes consideraciones de seguridad y efectividad. De igual forma, se puede decir que se han desarrollado cuatro categorías: blanqueamientos en la consulta aplicados por el profesional, prescritos por el odontólogo para uso ambulatorio, adquiridos por el usuario y aplicados por él mismo, y cualquier otra opción que no sea odontológica. ${ }^{18}$

Los clínicos regularmente recibirán niños y adolescentes con opacidades en el esmalte que afectan sus incisivos permanentes. Si causan preocupación al paciente, entonces se deben discutir las opciones de manejo apropiadas con el paciente, en el caso de niños y adolescentes, con sus padres o representantes, y se debe seleccionar y recomendar el tratamiento más apropiado basado en el estilo de vida del paciente, situación económica y salud bucal. ${ }^{18}$ La macroabrasión ${ }^{19}$ y la microabrasión están indicadas para las opacidades superficiales, mientras que el blanqueamiento puede tratar las opacidades profundas dentro del diente..$^{20}$

Blanqueamiento dental en niños y adolescentes, las sociedades científicas y el contexto legal.

La Asociación Latinoamericana de Odontopediatría (ALOP) recomienda el uso prudente de agentes blanqueadores para el paciente adolescente, realizando un examen detallado considerando la etapa del desarrollo, la higiene bucal del paciente y la presencia de lesiones de caries. El procedimiento debe realizarse con supervisión directa por parte del odontólogo para asegurar que sea el más eficaz y el menos invasivo posible, tomando en cuenta siempre los posibles efectos secundarios que se puedan presentar. ${ }^{21}$

Por su parte, la Academia Americana de Odontología Pediátrica (AAPD) reconoce el incremento en la demanda de procedimientos de blanqueamiento dental por niños y adolescentes, ${ }^{2,3}$ por lo que ha recomendado el uso supervisado y cuidadoso del blanqueamiento en dientes vitales y no vitales, la individualización de planes de tratamiento para determinar el momento y tipo de blanqueamiento más apropiado para el paciente, la consideración de efectos adversos y la realización de más estudios relacionados al blanqueamiento dental en niños y adolescentes.

La regulación de la Unión Europea en relación al blanqueamiento dental de octubre de 2012, señala que el peróxido de hidrógeno al $6 \%$ puede ser utilizado cuando el tratamiento sea supervisado directamente por un profesional de la odontología y no podrá ser usado por personas menores a los 18 años de edad. Posteriormente en 2014, el Consejo Dental General del Reino Unido permitió el uso de peróxido de hidrógeno entre $0,1 \%$ y $6 \%$ en pacientes menores de 18 años de edad solo para el tratamiento o prevención de una enfermedad o problema mayor. ${ }^{20,22}$ Así mismo, se han realizado publicaciones cuestionando esta posición, expresando que su uso se limita debido a una burocracia más que por la edad del paciente, e insta a utilizar mejor evidencia disponible para resolver este aspecto, y visualizar métodos menos destructivos para el paciente pediátrico. ${ }^{23}$ 
En concordancia, Walshaw et al. han señalado que el blanqueamiento dental es la única opción razonable de tratamiento en casos de dientes con cambio de coloración asociado a traumatismo dentario, considerando especialmente el efecto que la estética dental tiene en esta etapa difícil de desarrollo psicosocial. ${ }^{20}$

Así mismo, Donly et al. reportaron que el blanqueamiento en niños y adolescentes es un procedimiento seguro utilizando peróxido de carbamida en las noches y tiras de blanqueamiento de peróxido de hidrógeno durante un corto período de tiempo $^{24}$ por su bajo porcentaje del gel blanqueador. También, Croll y Donly concluyeron que los procedimientos de blanqueamiento dental que se realizan en adultos, pueden realizarse de manera segura en adolescentes supervisados por un adulto durante el proceso, ${ }^{25} \mathrm{y}$ correctamente indicado por el odontólogo. De igual forma, en 2016, se realizó un estudio clínico aleatorizado cuyo objetivo fue evaluar la eficiencia de dos sistemas de blanqueamiento en adolescentes haciendo un seguimiento de doce meses. En un grupo se utilizó peróxido de hidrógeno al $25 \%$ con luz ultravioleta y en el otro grupo se utilizó peróxido de hidrógeno al $36 \%$ con luz LED. El estudio demostró que ambos geles podían utilizarse de forma segura y efectiva en dentición permanente joven sin sensibilidad alguna reportada por parte de los pacientes, aunque establece que la recidiva se presentó con el gel de menor concentración en el término de tiempo evaluado. ${ }^{26}$

En países como el Reino Unido, el blanqueamiento dental a pesar de ser una opción de tratamiento segura y efectiva aún no está disponible para pacientes niños debido a problemas en la cobertura de seguros. Sin embargo, Walshaw et al. demostraron con tres casos que el blanqueamiento dental era la opción de tratamiento más adecuada. Eliminar esta opción de tratamiento para pacientes pediátricos seleccionados, generó preocupación a los clínicos tratantes, ya que se debían realizar tratamientos más invasivos y menos efectivos en detrimento de la salud bucal a corto y largo plazo. Además, el hecho de no proporcionar ningún tratamiento puede afectar negativamente la calidad de vida y el bienestar psicológico de los niños. ${ }^{22}$

\section{Efectos sobre la vitalidad pulpar}

Es importante comprender el mecanismo de acción de los agentes blanqueadores y las posibles interacciones con el tejido dentario para minimizar los efectos indeseables, como la hipersensibilidad dentinaria.

El peróxido de hidrógeno es un radical libre inestable de bajo peso molecular que penetra en el esmalte y la dentina a través de la difusión. Las moléculas de pigmentos orgánicos de los tejidos se rompen convirtiéndose en moléculas hidrofílicas simples mediante un proceso de óxidoreducción. Estas moléculas más simples son removidas del tejido dentario cuando entran en contacto con agua produciendo el efecto blanqueador deseado. Ambos sistemas de blanqueamiento (ambulatorio $\mathrm{y}$ en el consultorio) tienen el mismo mecanismo de acción. ${ }^{27}$

La respuesta pulpar es un aspecto importante a ser considerado en el blanqueamiento en adolescentes, ya que los componentes de los blanqueadores pueden difundir a través del diente $\mathrm{y}$ alcanzar el tejido pulpar causando un 
estrés oxidativo y efectos citotóxicos permanentes. Los radicales liberados como iones hidroxilos y oxígeno pueden causar la oxidación de la cadena de fosfolípidos de la membrana celular y el citoplasma lisosomal causando daño en el tejido pulpar que va desde estrés oxidativo hasta muerte pulpar. ${ }^{3,27}$

La saturación de oxígeno pulpar normal varía entre $75 \%$ y $92,60 \%$ y por debajo de $74,6 \%$ en pulpas necróticas. Esta saturación de oxígeno disminuye con la edad y el desarrollo dentario. ${ }^{28}$ Con el tratamiento de blanqueamiento en el consultorio se produce una reducción inmediata en el flujo sanguíneo y en consecuencia una reducción en la saturación de oxígeno. De nuevo, el estrés oxidativo que se produce en el tejido pulpar a consecuencia de la penetración del peróxido de hidrógeno puede dar origen a una respuesta inflamatoria, que se traduce en un aumento del flujo sanguíneo y permeabilidad vascular de los capilares pulpares. Debido a que el tejido pulpar se encuentra entre las paredes radiculares y solo tiene acceso a la vascularización a través del foramen apical, la presión intrapulpar aumenta rápidamente, disminuyendo el flujo sanguíneo, lo cual explica la reducción en la saturación de oxígeno que va aumentando progresivamente hasta alcanzar una elevación con relación a la línea base a los 30 días de seguimiento posteriores al procedimiento de blanqueamiento dental. Sin embargo, algunos estudios han señalado que la hipoxia sostenida durante 24 horas en el tejido pulpar es capaz de producir la interrupción del crecimiento celular e inducir la apoptosis, por lo que se recomienda vigilar la saturación de oxígeno durante el procedimiento dental y una vez más, controlar su libre uso. Así mismo es necesario señalar que los geles de peróxido de carbamida al 10\%, utilizados para tratamiento de blanqueamiento dental ambulatorio, producen los niveles de saturación de oxígeno más favorables durante el tratamiento de blanqueamiento dental. La aplicación por cortos períodos de tiempo y a baja concentración podrían minimizar los efectos tóxicos de los agentes blanqueadores sobre el tejido pulpar. ${ }^{29}$

\section{Blanqueamiento y la hipersensibilidad dental}

La sensibilidad dental es el efecto más indeseable del procedimiento y afecta entre el $8 \%$ y el $66 \%$ de los casos tratados con peróxido de hidrógeno, ${ }^{3}$ y del $15 \%$ al $65 \%$ de los pacientes que reciben tratamiento con peróxido de carbamida, ${ }^{20}$ normalmente con un grado moderado de dolor en las primeras etapas del tratamiento. Las hipótesis sugieren que la hipersensibilidad ocurre por la difusión de los agentes blanqueadores a través del esmalte y la dentina, alcanzando y lesionando al tejido pulpar. ${ }^{3,27,29}$ Algunos investigadores también han señalado que pueden formarse burbujas de oxígeno en los túbulos dentinarios que son capaces de causar movimiento en el fluido dentinario activando las terminaciones nerviosas. ${ }^{3}$

Adicionalmente, se ha reportado que la sensibilidad en los incisivos puede ser mayor debido a una menor cantidad de tejido duro dental, por lo que puede ser cuestionable utilizar geles de alta concentración en adolescentes, ya que esta población tiene un tejido dentinario de menor espesor cuando es comparada con la población adulta. Por esta razón, el tratamiento ambulatorio, que utiliza geles a menor concentración, pudiera ser el indicado para el paciente adolescente. Martin et al. compararon un gel al $6 \%$ 
de peróxido de hidrógeno y un gel de peróxido de carbamida al $35 \%$, observando que no existía diferencia en el efecto del blanqueamiento al mes de seguimiento, indicando que no es necesario usar geles de alta concentración. ${ }^{30}$ Así mismo el uso de tiras de peróxido de hidrógeno al 35\%, pueden producir más sensibilidad dental cuando son comparados con tratamientos ambulatorios, aun cuando se usen bajo supervisión profesional. ${ }^{27}$

La adición de compuestos de calcio puede reducir la sensibilidad. Estudios previos con fosfopéptidos de caseína con calcio y fosfato amorfo parecen incrementar la microdureza del esmalte en geles de baja concentración de peróxido de hidrógeno, sin alterar el efecto del agente blanqueador. $^{3}$ Sin embargo, estudios para analizar la efectividad de las pastas desensibilizantes, han señalado que estos productos no tienen efecto protector al ocluir los túbulos dentinarios pues no impiden la penetración del peróxido de hidrógeno en el tejido pulpar. ${ }^{29}$ Algunas pastas o geles desensibilizantes son analgésicas como el nitrato de potasio en distintas concentraciones, otras remineralizantes como el fluoruro de sodio y otras buscan ocluir esos túbulos como los oxalatos y barnices. De igual forma, los radicales libres difunden por aumento de la permeabilidad del tejido dentario más que por aumento de la luz de los túbulos.

A pesar de que se ha sugerido que las lámparas halógenas, láser o LED pueden acelerar el proceso de blanqueamiento, su efecto no ha podido ser clínicamente comprobado y adicionalmente pueden ser una fuente de calor elevando la temperatura pulpar y la difusión de componentes tóxicos a través de los tejidos duros aumentando la sensibilidad. .,20 $^{3}$ Además, es poca la efectividad al energizar los geles, existe alto riesgo de sensibilidad y efecto sobre la pulpa y aumenta el costo de los tratamientos innecesariamente.

Como muestra de los avances e investigaciones que se están produciendo en torno al blanqueamiento, inicialmente se ha demostrado que la incorporación de polímeros nanoparticulados ha mejorado la eficacia y estabilidad de los peróxidos de carbamida. Luego de las pruebas realizadas, los dientes extraídos no mostraron daño pulpar in situ en la evaluación histológica. Todo esto logró revelar un nuevo horizonte con un producto mas estable, lo cual es un mejor enfoque para el uso del peróxido de carbamida. ${ }^{31}$

De igual manera, Batista et al. analizaron la adición de gluconato de manganeso al $0,01 \%$ al gel de peróxido de carbamida al $10 \%$ para influir en su activación química disminuyendo el tiempo de uso e incrementando el grado de blanqueamiento. ${ }^{32}$

Tratamiento farmacológico de la hipersensibilidad dentaria post-operatoria

Son muchas las combinaciones de medicamentos que se han utilizado para el control de la sensibilidad derivada del blanqueamiento dental. Se han utilizado distintas posologías para prevenir la sensibilidad o en su defecto disminuir la intensidad de la misma. Rezende et al. analizaron los trabajos donde utilizaron dexametasona, un antinflamatorio a una dosis de $8 \mathrm{mg}$. 1 hora antes del procedimiento y dosis de $4 \mathrm{mg}$. cada 6 horas durante 48 horas, concluyendo que su efecto farmacológico no evitaba ni 
disminuía la sensibilidad dentaria, por lo que no sejustifica su utilización33. También Coppla et al. analizaron los estudios donde se usaban las combinaciones de un analgésico no opiode (acetaminofen/ paracetamol) y un analgésico opiode (codeína) a una dosis $500 \mathrm{mg} / 30 \mathrm{mg}$ respectivamente 1 hora antes y cada 6 horas durante 48 horas observando que esta combinación tampoco reduce el riesgo y la intensidad de la sensibilidad dentaria provocada por los blanqueamientos en los consultorios. ${ }^{34}$ De Oliveira et al. en un ensayo clínico aleatorizado compararon la eficacia del ibuprofeno y del acetaminofén/ paracetamol combinado con codeína para controlar la sensibilidad inmediata causada por el blanqueamiento dental en el consultorio, realizado con peróxido de hidrógeno al 35\%, concluyendo que el uso de la combinación de acetaminofén/ paracetamol mas codeína más 1 hora antes del blanqueamiento dental en el consultorio puede reducir drásticamentela intensidad de la sensibilidad inmediata. ${ }^{35}$

Por su parte, Faria et al. realizaron una revisión sistemática sobre el uso de los analgésicos antiinflamatorios no esteroideos (AINEs) donde se indicaban antes de realizar el blanqueamiento como analgesia preventiva y se repetía la dosis al finalizar, encontrándose que no hay un efecto significativo en la aparición y disminución de la sensibilidad, por lo que no estaría justificado su uso. ${ }^{36}$ Un resultado similar encontraron Vaez et al. donde la administración preventiva de una dosis única de $400 \mathrm{mg}$ de etodolaco no logró disminuir la sensibilidad dental durante o después del blanqueamiento realizado en el consultorio. ${ }^{37}$

En los estudios más recientes llevados a cabo por Almassri et al. en 2019 con el fin de evaluar la efectividad de medicamentos analgésicos antiinflamatorios para el control de la sensibilidad dental, no solo durante sino después de la realización de los blanqueamientos en pacientes adultos, se concluyó que no tienen un efecto clínicamente significativo. ${ }^{38}$

Se necesitan más estudios para saber si realmente existealguna combinación eficaz para evitar el problema de la sensibilidad post tratamiento con blanqueamientos $\mathrm{o}$ al menos reducir su intensidad. Porque como señala Brignardello, puede haber una pequeña reducción del dolor después del blanqueamiento dental al tomar acetaminofén/paracetamol combinado con codeína ya que este medicamento potencia el efecto del analgésico, pero es importante considerar los riesgos potenciales del consumo de un opiode. ${ }^{39}$

Blanqueamiento y efectos sobre tejidos blandos

El blanqueamiento no es un procedimiento sin riesgos, la ulceración gingival y labial están asociadas al mismo. Para reducir el riesgo de lesión gingival se debe realizar aislamiento absoluto durante el tratamiento en la consulta o en su defecto una barrera gingival fotocurada correctamente colocada y sellada, y se debe vigilar la extensión y ajuste de la cubeta para los procedimientos de blanqueamiento ambulatorio. El uso de tiras de peróxido de hidrógeno han sido asociadas a mayor queja de laceración gingival y labial, probablemente debido a que no son individualizadas, son productos de alta concentración y pueden tener contacto directo con los tejidos blandos. ${ }^{20}$ Para disminuir la irritación gingival, se recomienda el uso de vitamina E, $400 \mathrm{mg}$ antes y después del tratamiento en el consultorio. 
Blanqueamiento y productos sin prescripción profesional

La mayoría de los autores coinciden en señalar que el blanqueamiento favorece la autoestima del paciente. Los preceptos sociales han llevado a los fabricantes desde el año 2000, a producir productos de venta en farmacias y supermercados que no requieren la prescripción, ni la vigilancia del profesional y adicionalmente tienen un menor costo para el paciente. También se han incorporado a pastas dentales $y$ enjuagues bucales. Debido a lasdistintas concentraciones de peróxidos en estos productos, se tiene poca certeza de su efecto blanqueador. Además, el uso de éstos ha causado cierta inconformidad en la comunidad científica debido a la falta de supervisión profesional considerando el posible efecto nocivo, las alteraciones morfológicas, la sensibilidad, la posible ingesta y las reacciones alérgicas que pueden ocasionar los productos con altas concentraciones de agentes blanqueadores como es el caso de algunas tiras de blanqueamiento. La falta de supervisión profesional puede derivar en un uso inapropiado de estos productos. ${ }^{27}$

Es poco lo que se conoce de los efectos de estos productos que se publicitan con posibilidades de éxito y poca bioseguridad para el paciente.

Se ha publicado que estos geles mejoran las manchas dentarias extrínsecas y que producen blanqueamiento pero que el efecto cesa apenas se elimina su uso. Además, los autores sugieren más estudios que confirmen estos resultados. ${ }^{40}$ También se han realizado estudios para comparar la eficacia de este tipo productos blanqueadores (enjuagues y pastas dentales), pero han sido tan variados los tipos de diseño de ensayos, que hacen que las comparaciones directas sean difíciles, sobre todo porque hay varias formas de medir el color resultante, y no hay consenso de opinión en este campo. ${ }^{41}$ Incluso hasta se han comparado con la eficacia de tratamientos de blanqueamientos ambulatorios, ${ }^{42}$ lo cual dista mucho de la realidad.

Blanqueamiento y pacientes con defectos de esmalte

Pan et al. realizaron un estudio muy interesante donde evaluaron 22 pacientes con 186 dientes fluoróticos a los que se les indicó blanqueamiento ambulatorio y resinas infiltrantes. Los efectos del tratamiento se evaluaron mediante cuestionarios, incluidos los cambios en la evaluación subjetiva de los dientes y el estado psicológico de los pacientes antes y después de los tratamientos. Se tomaron fotografías digitales estandarizadas en cada momento del proceso de tratamiento, incluida la línea de base después del blanqueamiento, inmediatamente después del tratamiento y más de seis meses después del tratamiento. La mala apariencia de los dientes causó que el $13,64 \%$ de los pacientes a menudo estuviera deprimido, frustrado o decepcionado, mientras que el $72,72 \%$ ocasionalmente tenía estos sentimientos. Después del tratamiento, la satisfacción de los pacientes con respecto al aspecto dental aumentó de $0 \%$ (satisfecho) a 58,82\% (satisfecho) y 23,53\% (muy satisfecho). Además, estos tratamientos mejoraron la confianza de todos los pacientes en sonreír, reír y mostrar sus dientes. ${ }^{43}$

Un ensayo clínico controlado llevado a cabo por Schoppmeier et al. reveló que el blanqueamiento en el consultorio con 
peróxido de hidrógeno al 25\% antes de la resina infiltrativa proporciona efectos de enmascaramiento significativamente mejores en pacientes con fluorosis. Los resultados de este estudio sugieren que la infiltración de resina sola puede enmascarar eficazmente la fluorosis dental leve a moderada en adultos jóvenes, pero que con el blanqueamiento los resultados clínicos se potencian y mejoran. ${ }^{44}$

Para el tratamiento de las opacidades difusas se ha propuesto el blanqueamiento con peróxido de hidrógeno o peróxido de carbamida. Aun cuando en los reportes se han utilizado diferentes tipos de concentraciones, todos han logrado una mejoría estética.

También en una revisión sistemática y meta-análisis realizada en 2019 se concluyó que el blanqueamiento vital produce cambios positivos en la calidad de vida de los participantes jóvenes en aspectos como sonreír, reír y mostrar los dientes sin ninguna vergüenza. ${ }^{45}$

En caso de la Amelogénesis Imperfecta, esta condición provoca un gran sufrimiento en quienes la padecen, pues tiene repercusiones sociales y emocionales desde la niñez. ${ }^{46}$ Resulta comprensible que en la mente de estos pacientes se formen mecanismos de defensas para protegerse del ambiente hostil que los rodea y en forma evidente son rechazados por su grupo etario; estos mecanismos protectores están relacionados con: sumisión, agresión y retracción. En este último, los niños prefieren vivir en un mundo de fantasías que afrontar este entorno desagradable. Así sobrellevan su malestar psicológico que provoca el defecto dental; los sentimientos de vergüenza, culpa, ineptitud, indignidad, recibiendo burlas y sufrimiento como sucede con cualquier enfermedad crónica. ${ }^{47}$ Sin embargo, en la amelogénesis imperfecta no suele indicadarse el uso de blanqueamiento dental en la mayoría de los casos por presentar gran queja de sensibilidad, gran daño estructural, pérdida de estructura dentaria, enfermedad periodontal y considerables necesidades de rehabilitación que hacen que estos tratamientos sean poco útiles bajo estas condiciones.

\section{Blanqueamiento en niños con HMI}

El término Hipomineralización MolarIncisivo (HMI) se utiliza para definir un defecto cualitativo del desarrollo del esmalte dental, que tiene un origen sistémico y afecta a uno o más de los primeros molares permanentes con o sin afectación de los incisivos permanentes. Los defectos derivados de la HMI pueden influir en el estado de salud general y la calidad de vida de los niños afectados. La marcada hipersensibilidad que presentan la mayoría de los niños con HMI, empeora la higiene del paciente aumentando el riesgo de desarrollar lesiones de caries dental. La queja estética es frecuente debido a los defectos que se generan en los incisivos, lo cual repercute negativamente en la autoestima y autoimagen del paciente con HMI. ${ }^{48-50}$

En un estudio relacionado a la percepción del defecto por los odontólogos tratantes se encontró que la recuperación de la estética representa el segundo reto más referido por los profesionales $(20 \%)$, en el abordaje terapéutico de HMI, ocupando el primer lugar, la longevidad de las restauraciones $(26 \%) .{ }^{51}$

Considerando que los defectos marrones 
y amarillos se relacionan a lesiones más profundas y severas, es probable que las opacidades blancas y crema puedan responder satisfactoriamente a los procedimientos de blanqueamiento dental con peróxido de carbamida, así como a la macroabrasión y microabrasión con ácido clorhídrico o ácido fosfórico. En ocasiones debe recurrirse a una combinación de dos técnicas, microabrasión y blanqueamiento. Los tratamientos conservadores deben ser considerados antes de instaurar tratamientos restauradores e invasivos pues debe tomarse en cuenta las posibles complicaciones derivadas de la extensión del defecto hasta el tejido pulpar y el contorno gingival de los incisivos en la dentición permanente joven. Adicionalmente es necesario considerar que las opacidades de los incisivos, a menudo suelen hacerse menos profundas a largo plazo. ${ }^{19,52,53}$

También es interesante señalar que en una revisión sistemática y en estudios de percepción y conocimientos en relación con la HMI, los odontólogos tratantes parecen inclinarse por el uso de resinas infiltrantes y la microabrasión para el tratamiento de las opacidades de los incisivos afectados, ${ }^{54,55}$ lo cual puede comprobar que los odontólogos tienen poco conocimiento de los alcances del blanqueamiento, o no conocen muchas alternativas en relación a este tratamiento.

Es bajo estas condiciones que se deben analizar las múltiples ofertas y concentraciones de peróxido de carbamida que existen en el mercado, de esta forma, se puede ofrecer un tratamiento no invasivo para mejorar la presencia de las opacidades incluso si se va a restaurar ya que puede minimizar el posible desgaste necesario y optimizar las bondades de los materiales estéticos.
Blanqueamiento, calidad de vida, impacto psicosocial

Considerando que la apariencia tiene un fuerte impacto en la población adolescente y se ha relacionado con un desarrollo social positivo, es importante determinar el nivel de satisfacción del blanqueamiento, también porque se trata de un procedimiento electivo. Algunos estudios han señalado que los adolescentes pueden mostrarse satisfechos entre la segunda y sexta semana del procedimiento de blanqueamiento dental independientemente del tipo de procedimiento utilizado. ${ }^{27}$

La calidad de vida es compleja y abarca diferentes dominios. Si bien seha detectado un impacto positivo del blanqueamiento dental, también se han informado dificultades en la higiene dental y el dolor resultante del tratamiento, y esto puede afectar negativamente el rendimiento diario. ${ }^{56}$

\section{Discusión}

El blanqueamiento dental es sin duda la alternativa de tratamiento más conservadora que puede instaurarse en un adolescente con queja estética, derivada de una elevada demanda de su apariencia física o de la presencia de defectos de desarrollo del esmalte en los dientes expuestos en la línea de la sonrisa. ${ }^{51,52,56}$

Es necesario señalar que en algunos países las prohibiciones legales pueden restringir la realización de dichos procedimientos en niños y adolescentes, por lo que el profesional deberá determinar las posibles complicaciones psicosociales que pueden 
derivarse de la situación clínica, para tomar la decisión terapéutica adecuada en cada caso, haciendo un balance entre los beneficios y las posibles complicaciones y efectos adversos. ${ }^{20,22,58-60}$

Contradictoriamente los productos de blanqueamiento que se ofrecen de venta libre y sin prescripción profesional, fueron introducidos en el mercado desde el año $2000^{61,62}$ y aun cuando tienen algún efecto blanqueador, poseen altas concentraciones de peróxido de hidrógeno y pueden causar daño a los tejidos bucales cuando son utilizados sin supervisión profesional. Algunos estudios han demostrado que producen más sensibilidad dental y lesiones del tejido blando y gingival que los productos de uso profesional, ${ }^{27}$ por esta razón es de fundamental importancia que tanto los padres como el paciente adolescente reciban la oportuna indicación profesional para evitar el uso de productos que puedan resultar perjudiciales en la salud del paciente.

Se ha demostrado que los geles de baja concentración $(10 \%)$ de peróxido de carbamida utilizados en los tratamientos ambulatorios producen el mismo efecto blanqueador que los geles de alta concentración de peróxido de hidrógeno utilizados en el consultorio dental. Los primeros se consideran el tratamiento de primera elección para el paciente adolescente pues los productos de baja concentración tienen una menor posibilidad de difundir a través del esmalte y la dentina, reduciendo los efectos adversos sobre el tejido pulpar, son más efectivos, más predecibles y sus

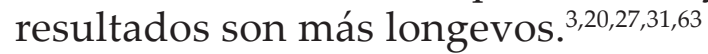

Los niños pequeños no son muy receptivos con los procedimientos de blanqueamiento ambulatorio por el tiempo de uso, es por esta razón que se pueden indicar peróxidos de hidrógeno al $6 \%$ de forma siempre ambulatoria por media hora a una hora con cubetas individualizadas. Estos no presentan diferencias significativas en comparación con los peróxidos de hidrógeno al 35\% en relación con su efectividad, además no generar hipersensibilidad dentinaria. ${ }^{64}$

Es necesario considerar que, en comparación con los adultos, el paciente adolescente aun cuando puede verse más motivado a cumplir con el tratamiento ambulatorio, también puede abandonarlo si no observa el efecto deseado a corto plazo, por lo que deberán evaluarse previamente las expectativas del paciente y los padres, para evitar fracasos innecesarios haciendo más predecibles los objetivos. Son indispensables la adecuada confección de la cubeta, la individualización del protocolo y el apropiado seguimiento profesional para garantizar el éxito del tratamiento. ${ }^{65}$ Sin embargo, es imperativo realizar estudios clínicos de seguimiento en la población adolescente para determinar las ventajas y desventajas de los productos de blanqueamiento de uso profesional y sus protocolos.

También debe tomarse en cuenta que el uso de fármacos analgésicos antes o después del blanqueamiento, no ha mostrado un efecto clínicamente significativo $^{34,36-38}$ siendo necesarios más estudios a fin de determinar cuál es el medicamento más eficaz para el control de la sensibilidad, ya que es uno de los motivos más comunes para la interrupción del tratamiento ${ }^{38}$. La combinación de acetaminofén/paracetamol con codeína 
1 hora antes del blanqueamiento dental realizado en el consultorio ha resultado ser la más efectiva para reducir la intensidad de la sensibilidad inmediata, ${ }^{35}$ pero esta combinación no debe utilizarse en el paciente pediátrico debido al riesgo que conlleva la ingesta de codeína en ciertos pacientes que son metabolizadores extensos o ultrarápidos de codeína, que es una condición genética donde la codeína se transforma en morfina más rápidamente y por tanto tendrán más posibilidades de presentar reacciones adversas derivadas de la intoxicación de morfina. El porcentaje de niños con esta condición es variable, dependiendo de su origen étnico y no existe ninguna prueba disponible en la práctica clínica que permita conocer el grado de actividad enzimática. ${ }^{66}$

Por otro lado, merecen especial atención los niños y adolescentes con opacidades relacionadas a defectos del desarrollo del esmalte dental, con queja estética derivada de la afectación de dientes expuestos y mostrados durante la sonrisa o el habla. Se ha propuesto el blanqueamiento dental como alternativa para aliviar el contraste producido por estas opacidades y el esmalte sano, sin embargo, es necesario enfatizar que los autores coinciden en señalar que los procedimientos de blanqueamiento dental parecen no corregir por si solos los defectos visibles en la superficie dental (aunque lo mejoran sustancialmente), por lo que en algunas oportunidades deben combinarse con otras técnicas como microabrasión, resinas infiltrantes o restauraciones. ${ }^{43,44,53}$

El clínico deberá determinar con mucha cautela el tratamiento indicado en cada uno de los casos de opacidades, pues el diagnóstico del defecto tiene suprema importancia, considerando que las alteraciones de la estructura dental pueden tener respuestas muy distintas a los diferentes tipos de tratamiento, derivadas de las características histológicas, químicas y estructurales de cada condición. Si bien, como ya se expresó, el blanqueamiento puede disminuir el contraste entre la mancha y el resto del tejido dentario cuando es correctamente indicado y efectivamente logrado. Puede no resolver completamente la visibilidad del defecto del esmalte, pero sí disminuye su presencia al observador e inclusive mejora las probabilidades de una restauración con calidad estética y menos invasiva.

Es necesario destacar que algunos autores han encontrado un efecto altamente beneficioso y recomiendan el uso de productos a base de fosfopéptidos de caseína concalcioy fosfatoamorfo ${ }^{67}$ durante el procedimiento de blanqueamiento dental en casos de opacidades dentarias. Otros investigadores no han encontrado un efecto clínico significativo con el uso de pasta desensibilizantes ${ }^{29}$ y geles blanqueadores con calcio ${ }^{3}$ añadido en adolescentes sin diagnóstico de defectos de esmalte, por lo que se requiere dar instrucciones detalladas para evitar falsas expectativas en relación con el alivio de la sensibilidad dental.

Así mismo, considerando las complicaciones que pueden presentarse en los dientes de niños pequeños con un espesor reducido de tejidos duros y amplio contenido pulpar, el tratamiento de las opacidades con procedimientos de blanqueamientodental puedeconsiderarse como una alternativa segura hacia el final de la segunda fase de dentición mixta, o durante la adolescencia, momento en el cual los pacientes suelen empezar a manifestar su preocupación por el aspecto 
físico. ${ }^{52}$ La misma recomendación deberá hacerse para aquellos niños que reporten una elevada queja estética, derivada del llamativo contraste de color entre la dentición permanente de aspecto más amarillento y la dentición primaria de aspecto muy blanco. Esta situación clínica es un motivo de consulta frecuente durante la primera fase de dentición mixta.

Cualquier defecto dentario provoca cierto malestar emocional en los pacientes que lo padecen y manifiestan aspectos emocionales, tales como: rechazo a sí mismo, no se aceptan ni se sienten satisfechos consigo mismo. ${ }^{68} \mathrm{El}$ impacto de los defectos visibles tiene efectos negativos en la autopercepción ${ }^{69}$ conviniendo que un autoconcepto negativo de sí mismo acentúa la percepción del defecto. ${ }^{13}$

El blanqueamiento puede percibirse como un recurso válido para restaurar la autoimagen en los pacientes; apoyados en Nair et al. ${ }^{70}$ y Nagashree et al. ${ }^{71}$, quienes afirman que las opacidades difusas severas son desagradables en comparación con las leves y moderadas. Teniendo en cuenta que cuando una persona se percibe a sí misma con algún defecto, requerirá asumir con justa medida la preocupación que existe en torno a su aspecto físico, cuando la belleza es algo tan subjetivo. Ante esto, la condición que padecen estos pacientes es de gran relevancia también para la familia o personas que lo rodean. Por tanto, se hace necesario retomar el camino que lleva al adolescente hacia la recuperación de la autoconfianza y autoimagen acordes a lo que ofrece la estética dental. Corroborando, la evidencia científica indica que en la pubertad el cerebro es particularmente susceptible al estrés ${ }^{72}$ mientras que adultos expuestos a un mismo estrés repetidamente se habitúan a él, los puberales siguen mostrando picos de corticosterona más altos en la edad adulta $^{73}$ lo que favorece la vulnerabilidad a diferentes tipos de enfermedades. ${ }^{74}$

Por consiguiente, el blanqueamiento como tratamiento dental estético desde la edad temprana cobra un valor especial en niños y jóvenes y se puede decir que tiene efectos positivos en el desarrollo psicosocial del individuo, en vista de que favorece la atracción física y la seguridad emocional que necesita toda persona al sonreír y por ende facilita la socialización ${ }^{75}$ y sobresalen sus beneficios en la pubertad. La prevención de enfermedades que se relacionan con el manejo del estrés indica la importancia de ayudar a los jóvenes a quererse a sí mismo, aceptarse e ir con confianza ante la vida.

Está claro que el blanqueamiento dental viene a sumar en gran medida al alivio de las afecciones estéticas de los niños y adolescentes, otorgando confianza y seguridad a quien lo obtiene, con una suma de factores positivos en la percepción de sí mismo. Su indicación correcta, oportuna y bajo consideraciones de un sólido equipo multidisciplinario, entrega múltiples beneficios en contraposición a los efectos adversos que se pueden presentar como la hipersensibilidad dentinaria. Se debe realizar un control cercano del progreso y cumplimiento del tratamiento en todo paciente.

Los sistemas de blanqueamiento han demostrado en muchos sentidos su eficacia y hasta cierto punto su bioseguridad. Su uso se ha generalizado y el logro de los objetivos que se proponen los clínicos es alcanzado con una máxima conservación de tejidos sanos. Los efectos secundarios son minimizados y controlados con los avances en el desarrollo de distintos materiales y las evaluaciones de nuevos 
protocolos con posibilidad de éxito. Las diferentes concentraciones de peróxidos de carbamida ofrecidas por los fabricantes, permiten una diversidad de alternativas y aún mayor control del procedimiento con muy bajo índice de efectos indeseables. Por todos los argumentos expuestos, se recomienda considerar las distintas indicaciones en pacientes pediátricos $\mathrm{y}$ adolescentes y extender su uso con el fin de mejorar los problemas no solo estéticos que se puedan presentar, sino también minimizar la necesidad de restauraciones tempranas, evitar la desinserción social y la deserción escolar. Falta mucho por investigar en relación al alcance de los blanqueamientos dentales, se esperan avances en su composición química y formulaciones, uso de nuevos catalizadores y nuevas investigaciones para lograr afianzar en el futuro las grandes posibilidades de estos sistemas.

\section{Referencias bibliográficas}

1. Corby P, Biesbrock A, Gorlach R, Corby A, Moreira A, Schork N, Bretz W. Treatment Responses to Tooth Whitening in Twins. Twin Res Hum Genet. 2014;17(1):23-6.

2. American Academy of Pediatric Dentistry. Policy on the Use of Dental Bleaching for Child and Adolescent Patients. 2019. https://www.aapd.org/globalassets/media/policies_guidelines/p_bleaching.pdf

3. Goncalvez ML, Tavares AC, Mota AC, Penna LA, Deana AM, Bussadori SK. In-Office Tooth Bleaching for Adolescents Using Hydrogen Peroxide-Based Gels: Clinical Trial. Braz Dent J. 2017;28(6):720-25.

4. Chen L, Zhang Q, Wang Y, Zhang K, Zou J. Comparing dental plaque microbiome diversity of extrinsic black stain in the primary dentition using Illumina MiSeq sequencing technique. BMC Oral Health. 2019 Dec 3;19(1):269.

5. Slots J. The microflora of black stain on human primary teeth. Scand J Dent Res. 1974;82(7):484-90.

6. Saba C, Solidani M, Berlutti F, Vestri A, Ottolenghi L, Polimeni A. Black stains in the mixed dentition: a PCR microbiological study of the etiopathogenic bacteria. J Clin Pediatr Dent. 2006;30(3):219-24.

7. Zhang F, Li Y, Xun Z, Zhang Q, Liu H, Chen F. A preliminary study on the relationship between iron and black extrinsic tooth stain in children. Lett Appl Microbiol. 2017;64(6):424-9.

8. Naranjo Sierra M. Terminología, clasificación y medición de los defectos en el desarrollo del esmalte. Revisión de literatura / Terminology, Classification and Measuring of the Developmental Defects of Enamel. Literature Review. Rev Jave. 2013;32(68):33-4.

9. Elfrink MEC, ten Cate JM, Jaddoe VWV, Hoffman A, Moll HA, Veerkamp JSJ. Deciduous Molar Hypomineralization and Molar Incisor Hypomineralization. J Dent Res. 2012;91(6):551-65.

10. Alaluusua S. Defining Developmental Enamel Defect-associated Childhood Caries: Where Are We Now?. J Dent Res. 2012;91(6):525-7.

11. Opydo-Szymaczek J, Gerreth K. Etiological factors related to dental fluorosis among children in Poznan, Poland- A preliminary Report. Fluoride. 2012;45(4):337-42.

12. Garg N, Jain AK, Saha S, Singh J. Essentiality of Early Diagnosis of Molar Incisor Hypomineralization in Children and Review of its Clinical Presentation, Etiology and Management. Int J Clin Paediatr Dent. 2012;5(3):190-6.

13. Marshman Z, Gibson B, Robinson Z, Gibson B, Robinson PG. The impact of developmental defects of enamel on young people in the UK. Commun Dent Oral Epidemiol. 2009;37:45-57.

14. Parekh S, Almehateb M, Cunningham SJ. How do children with amelogenesis imperfecta feel about their teeth? Int J Paediatr Dent. 2014;24:326-35.

15. Rodd HD, Marshman Z, Gibson B, et al. Oral health-related quality of life of children in relation to dental appearance and educational transition. Br Dent J. 2011;22:E4.

16. Craig SA, Baker SR, Rodd HD. How do children view other children who have visible enamel defects? Int J Paediatr Dent. 2015;25:399-408.

17. Dubey A, Avinash A, Bhat SS, Baliga MS. Twinkling stars: literature review on dental whitening in children. Indian J Dent Res Rev. 2012;Apr-Sep:1-3. 
18. Tooth Whitening/Bleaching: Treatment Considerations for Dentists and Their Patients. ADA Council on Scientific Affairs September 2009

19. Natera AE, Da Silva A, Benitez I, Moreno Y. TF4. Macroabrasión y Microabrasión del esmalte, ¿Es la secuencia correcta para resolver el problema de fluorosis dental? Reporte de un caso clínico Índice TF4. Rodyb. 2018;7(2):18-24.

20. Wallace A, Deery C. Management of Opacities in Children and Adolescents. Dental Update. 2015; 42(10):951-4.

21. Walshaw EG, Kandiah P, Rodd H. A triology of tragedies- Paediatric Dental Tooth Whitening. Br Dent J. 2019;227(11):959-60

22. Manual de la Revista Latinoamericana de Odontopediatría ALOP. 2da edición https://www. revistaodontopediatria.org/publicaciones/manuales/referencia-para-procedimientos-enodontopediatria-2da-edicion/Manual-de-Referencia-para-Procedimientos-en-Odontopediatria-2daedicion.pdf

23. Kelleher M. This law is an ass: legal and ethical issues surrounding the bleaching of young patients' discoloured teeth. FDJ.2014;5(2):56-67.

24. Donly KJ, Kennedy P, Segura A, Gerlach RW. Effectiveness and safety of tooth bleaching in teenagers. Paediatr Dent. 2005;27:298-302

25. Croll TP, Donly KJ. Tooth bleaching in children and teens. J Esthet Restor Dent 2014;26:147-50.

26. Bacaksiz A, Tulunoglu O, Tulunoglu I. Efficacy and Stability of Two in-Office Bleaching Agents in Adolescents: 12 Months Follow-Up. J Clin Pediatr Dent. 2016;40(4):269-73.

27. Pinto MM, de Godoy CH, Bortoletto CC, Olivan SR, Motta LJ, Altavista O, Lumi K, Sobral AP, Bussadori SK. Tooth Whitening With Hydrogen Peroxide in Adolescents: Study Protocol for a Randomized Controlled Trial. Trials. 2014;14(15):395

28. Bargrizan M, Asna M, Ahmadi M, Ramezani J. The Use of Pulse Oximetry in Evaluation of Pulp Vitality in Immature Permanent Teeth. Dent Traumatol. 2016;32(1):43-7.

29. Ferreira L, Goncalves A, Almeida D, Negro I, Zaiden M, Branco F, Estrela C. Effect of dental bleaching on pulp oxygen saturation in maxillary central incisors - a randomized clinical trial. J Appl Oral Sci. 2019;27:e20180442.

30. Martin J, Vildósola P, Bersezio C, Herrera A, Bortolatto J, Saad JR, et al. Effectiveness of $6 \%$ hydrogen peroxide concentration for tooth bleaching - A double-blind, randomized clinical trial. J Dent. 2015;43(8):965-72.

31. Lima FV, Mendes C, Zanetti-Ramos BG, et al. Carbamide peroxide nanoparticles for dental whitening application: Characterization, stability and in vivo/in situ evaluation. Colloids Surf B Biointerfaces. 2019;179:326-33.

32. Batista GR, Barcellos DC, Torres CR, Goto EH, Pucci CR, Borges AB. The influence of chemical activation on tooth bleaching using 10\% carbamide peroxide. Operative Dentistry, 2011, 36-2, 162-8.

33. Rezende M, Bonafé E, Vochikovski L, Farago PV, Loguercio AD, Reis A, Kossatz S. Pre- and postoperative dexamethasone does not reduce bleaching-induced tooth sensitivity: A randomized, triple-masked clinical trial. J Am Dent Assoc. 2016; 147(1):41-9.

34. Coppla FM, Rezende M, de Paula E, Farago PV, Loguercio AD, Kossatz S, Reis A. Combination of Acetaminophen/Codeine analgesics does not avoid bleaching-induced tooth sensitivity: a randomized, triple-blind two-center clinical trial. Oper Dent. 2018; 43(2):53-63.

35. De Oliveira SN, de Assunção IV, Borges BCD. Efficacy of ibuprofen and codeine + paracetamol to reduce immediate bleaching sensitivity caused by in-office tooth bleaching: A randomized, controlled, doubleblind clinical trial. Am J Dent. 2018;31(4):195-8.

36. Faria-E-Silva AL, Nahsan FP, Fernandes MT, Martins-Filho PR. Effect of preventive use of nonsteroidal anti-inflammatory drugs on sensitivity after dental bleaching: a systematic review and meta-analysis. J Am Dent Assoc. 2015; 146(2):87-93

37. Vaez SC, Faria-E-Silva AL, Loguércio AD, Fernandes MTG, Nahsan FPS. Preemptive use of etodolac on tooth sensitivity after in-office bleaching: a randomized clinical trial. J Appl Oral Sci. 2018; 1(26):e20160473.

38. Almassri HNS, Zhang Q, Yang X, Wu X. The effect of oral anti-inflammatory drugs on reducing tooth sensitivity due to in-office dental bleaching: A systematic review and meta-analysis. J Am Dent Assoc. 2019; 150(10):145-57.

39. Brignardello-Petersen R. There may be a small reduction in pain immediately after tooth bleaching when consuming codeine plus acetaminophen, but this is unlikely to be important to patients or worth the potential risks. J Am Dent Assoc. 2019;150(3):e35.

40. Ghassemi A, Hooper W, Vorwerk L, Domke T, DeSciscio P, Nathoo S. Effectiveness of a new dentifrice with baking soda and peroxide in removing extrinsic stain and whitening teeth. J Clin Dent. 2012;23(3):86-91 
41. Pontefract H, Sheen S, Moran J. The benefits of toothpaste - real or imagined? Review of its role in tooth whitening. Dent Update. 2001;28(2):67-70, 72, 74.

42. Torres CR, Perote LC, Gutierrez NC, Pucci CR, Borges AB. Efficacy of mouth rinses and toothpaste on tooth whitening. Oper Dent. 2013;38(1):57-62.

43. Pan Z, Que K, Liu J, Sun G, Chen Y, Wang L, Lou Y, Zhao M. Effects of at-home bleaching and resin infiltration treatments on the aesthetic and psychological status of patients with dental fluorosis: A prospective study. J Dent. 2019;91:103228

44. Schoppmeier CM, Derman SHM, Noack MJ, Wicht MJ. Power bleaching enhances resin infiltration masking effect of dental fluorosis. A randomized clinical trial. J Dent. 2018;79:77-84.

45. Kothari S, Gray AR, Lyons K, Tan XW, Brunton PA. Vital bleaching and oral-health-related quality of life in adults: A systematic review and meta-analysis. J Dent. 2019;84:22-9.

46. Montalvo A., Cabrera B., Quiñones S. Enfermedad crónica y sufrimiento: revisión de literatura. Aquichan. 2012. 12(2); 134-43

47. Rodríguez D, Acosta de Camargo MG, Pier-Doménico B, Tortolero MB. La Amelogénesis Imperfecta tratada por un equipo multidisciplinario. Reporte de un caso. Revista Oral-México 2010; 11(33):569-72

48. Jälevik B, Kilngberg G. Treatment outcomes and dental anxiety in 18 years-olds with MIH, comparisons with healthy controls- a longitudinal study. Int J Clin Pediatr Dent. 2012;22: 85-91.

49. Oyedele AT, Folayan MO, Adekoya-Sofowora CA, Oziegbe EO. Co-morbidities associated with molarincisor hypomineralisation in 8 to 16 year old pupils in Ile-Ife, Nigeria. BMC Oral Health. 2015;15:37.

50. Dantas-Neta NB, Moura LF, Cruz PF, Moura MS, Paiva SM, Martins CC, Lima MD. Impact of molarincisor hypomineralization on oral health-related quality of life in school children. Braz Oral Res. 2016;30(1):e117.

51. Upadhyay S, Kumar G, Dhilon J, Gill N. Perception of Indian Dental Surgeon Regarding Molar Incisor Hypomineralization. Int J Clin Ped Dent 2018;11(2):116-121.

52. Lygidakis NA, Wong F, Jälevik B, Vierrou A-M, Alaluusua S, Espelid I. Best Clinical Practice Guidance for clinicians dealing with children presenting with Molar-Incisor-Hypomineralisation (MIH) An EAPD Policy Document. Eur Arch Paediatr Dent. 2010;11(2):75-81.

53. Ghanim A, Silva MJ, Elfrink MEC, Lygidakis NA, Mariño RJ, Weerheijm KL, Manton DJ. Molar incisor hypomineralization (MIH) training manual for clinical field surveys and practice. Eur Arch Paediatr Dent. 2017;18(4):225-42.

54. Gamboa G, Lee G, Ekambaram M, Yiu C. Knowledge, perceptions, and clinical experiences on molar incisor hypomineralization among dental care providers in Hong Kong. BMC Oral Health 2018;13(1):217.

55. Alanzi A, Faridoun A, Kavvadia K, Ghanim A. Dentists' perception, knowledge, and clinical management of molar-incisor-hypomineralization in Kuwait: a cross-sectional study. BMC Oral Health. 2018;18(1):34.

56. Meireles SS, Goettems LM, Dantas RV, Bona ÁD, Santos IS, Demarco FF. Changes in oral health related quality of life after dental bleaching in a double-blind randomized clinical trial. J Dent. 2014;42(2):114-21.

57. Heymann HO, Swift EJ, Bayne SC, et al. Clinical evaluation of two carbamide peroxide tooth-whitening agents. Compend Contin Educ Dent 1998;19: 359-374.

58. American Academy of Pediatric Dentistry. Policy on the Use of Dental Bleaching for Child and Adolescent Patients. 2019. https://www.aapd.org/globalassets/media/policies_guidelines/p_bleaching.pdf

59. Carvalho LD, Bernardon JK, Bruzi G, Andrada MA, Vieira LC. Hypoplastic Enamel Treatment in Permanent Anterior Teeth of a Child. Operativ Dent. 2013; 38(4): 363-8.

60. Nagaveni NB, Umashankara KV, Radhika NB. Management of tooth discoloration in non-vital endodontically treated tooth - A report of 6 year follow-up. J Clin Exp Dent 2011;3(2):e180-3

61. Auschill TM, Savio TSD, Hellwig E, Arweiler NB. Randomized clinical trial of the efficacy, tolerability, and long-term color stability of two bleaching techniques: 18-month follow-up. Quintess Int. 2012;43(8):68393.

62. Lee S, Zhang W, Lee DH, Li Y. Tooth Whitening in Children and Adolescents: A Literature Review. Pediatr Dent. 2005; 27(5): 362-8

63. Chávez O, Rojas R, Márquez S. Efectividad de los procedimientos para el blanqueamiento dental. Una revisión sistemática. Rev Venez Invest Odont IADR 2016; 4(2): 122-60.

64. Martín J, Vildósola P, Bersezio C, Herrera A, Bortolatto J, Saad JR, Oliveira Jr OB, Fernández E. Effectiveness of $6 \%$ hydrogen peroxide concentration for tooth bleaching-A double-blind, randomized clinical trial. J Dent.2015;43(8):965-72.

65. Monteiro J, Ashley PF, Parekh. Vital bleaching for children with dental anomalies: EAPD members' survey. Eur Arch Paed Dent. 2019;https://doi.org/10.1007/s40368-019-00494-w 
66. Tobias JD, Green TP, Coté CJ. Section on anesthesiology and pain medicine. Committee on drugs. Codeine: Time to say “No" Pediatrics. 2016;138(4):e20162396.

67. Mastroberardino S, Campus G, Strohmenger L, Villa A, Cagetti MG. An Innovative Approach to Treat Incisors Hypomineralization (MIH): A Combined Use of Casein Phosphopeptide-Amorphous Calcium Phosphate and Hydrogen Peroxide- A Case Report. Case Rep Dent. 2012;2012:379593.

68. Tortolero MB, Acosta de Camargo MG, Natera A. Evaluación de la autoestima y la autoimagen en un grupo de niños de 6 a 11 años con defectos de esmalte. Rev Rodyb. 2019; 8(3):

69. Sneller J, Buchanan H, Parekh S. The impact of amelogenesis imperfecta and support needs of adolescents with AI and their parents: an exploratory study. International Journal of Pediatric Dentistry. 2014. 24(6); 409-16.

70. Nair R, Chuang JCP, Lee PSJ, Leo SJ, Yang NQ, Yee R, et al. Adult perceptions of dental fluorosis and select dental conditions-an Asian perspective. Community Dent Oral Epidemiol 2016; 44:135-44.

71. Nagashree SR, Shankar Aradhya MR y Arunadevi M. An investigation of social judgments made by young adults toward appearance of dental fluorosis. Indian J Dent Res. 2012;23(4):443-6.

72. Holder MK, Blaustein JD. Puberty and adolescence as a time of vulnerability to stressors that alter neuro behavioral processes. Front Neuroendocrinol 2014 35: 89-110.

73. Romeo RD, Bellani R, Karatsoreos IN, Chhua N, Vernov M, Conrad CD, McEwen BS. Stress history and pubertal development interact to shape hypothalamic-pituitary-adrenal axis plasticity. Endocrinology. 2006;147:1664-74.

74. Pagliaccio D, Luby JL, Bogdan R, Agrawal A, Gaffrey MS, Belden AC, Botteron KN, Harms MP, Barch DM. HPA axis genetic variation, pubertal status, and sex interact to predict amygdala and hippocampus responses to negative emotional faces in school-age children. Neuroimage. 2015;109:1-11.

75. Murillo K, Gamboa L, Morales F, Meza A, López A. Impacto emocional y en calidad de vida de individuos afectados por amelogénesis imperfecta. ODOVTOS-Int J Dental Sc. 2015;17(3):73-85.

Recibido: : 29/06/2020

Aceptado: 22/10/2020

Correspondencia: Maria Gabriela Acosta de Camargo, correo: macosta@uc.edu.ve 